\title{
Prolapsus de la Muqueuse gastrique à travers le Pylore
}

Cette malformation, connue de longue date - elle aurait été décrite par v. Schmieden en 1911 est Tobjet actuellement d'un grand inté-rêt, qui se traduit par de nombreux travaux: Manning et Híghsmíth ajoutaient 16 cas personnels aux 37 déjà publiés avant 1948. Ces dernières années, les travaux à ce sujet se multiplient, sans qu'on ait réussi à faire Гunanimité sur plusieurs points litigieux.

Sa frequence tout d'abord: on le rencontrerait plus souvent que $\Gamma$ ulcère, dit Melamed. Les proportions établies sur de longues series sont de 3,38\% (Cove et Curphrey) à 7,7\% (Ferguson). Bockus, dans son traité classique, admet que le prolapsus est «probablement frequent», mais reste latent aussi longtemps qu'il n'y a pas complication. Scott soutient $\Gamma$ opinion inverse, et affirme ne l'avoir jamais rencontre à $\Gamma$ examen radiologique systématique de 250 individus nondyspep-tiques. Les auteurs sont d'accord en revanche sur la forte predominance du sexe masculin, et sur $\Gamma$ apparition du prolapsus chez des adultes jeunes.

La clíníque ne permet guère de reeonnaître, ou même de soup-çonner le glissement de la muqueuse. Avec une precision très améri-caine, Wilson et Granges notent:

des «signes de dyspepsies» dans 20 cas sur 25; les douleurs, post-prandiales tardives, sont moiiis régulières que celles de $\Gamma$ ulcère, rare-

228

\section{Editorial}

ment nocturnes, et pas calmées par les medicaments antacides (la chlorhydrie du sue gastrique est rarement élevée);

les vomissements, «surtout après des rep as volumineux», sont signalés 12 fois sur 25; ils

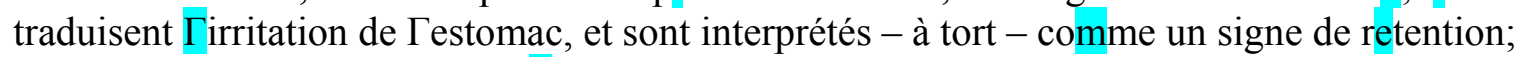
enfin la muqueuse, repliée sur elle-même, tend à saigner ( $28 \%$ des observations), et les hémorragies sont souvent massives, comme dans les observations de Bralow et coll.

Ces troubles durent en general longtemps - une à six années -avant d'etre reconnus. Les examens physiques (et même psychia-triques, dit van Noatel) n'aident pas à la comprehension de ces états.

La clinique n'apporte done rien de caractéristique, et e'est la radíologíe qui fournit les elements du diagnostic, depuis les descriptions de Elíason, Pendergrass et Wright (1926). Dans une solide etude récente - la premiere dans notre pays - Zimmer (de Fribourg) analyse de façon magistrale Гanatomie pathologique de la lesion et de sa traduction sous les rayons X. Le prolapsus peut être total, circulaire: toute la muqueuse est alors évaginée à travers le pylore et forme, à la base du bulbe, l'image typique en ombrelle. Dans le prolapsus partíel, il s'agit du déplacement d'un ou plusieurs plis de la muqueuse, mais la lesion reste unilatérale, donnant alors ces aspects lacunaires ou en «demi-champignons», plus difficiles à interpreter; on les prend parfois pour un polype ou un cancer. De magnifiques cliches, expliqués par de clairs schémas, illustrent le mémoire de Zimmer et fournissent de beaux exemples de ces diverses formes. Ferguson insiste encore sur deux particularités radiologiques: la tolerance au baryum du bulbe, qui ne paraît pas 
irrité, et se laisse parfaitement remplir de substance opaque; Гinconstance du prolapsus, qui en station verticale, échappe notamment à robservation.

Les hypotheses pathogéníques ne sont guère satisfaisantes: chacun s'accorde à reconnaitre Гinflammation et Thypertrophie prépylo-riques; mais évoquer une «predisposition locale», une tendance au glissement par manque de rigidité de la muqueuse, ou faire intervenir un «facteur neurogénique», ne nous éclaire guère. Une autre explication est franchement paradoxale: le prolapsus surviendrait dans les hypertrophies pyloriques, comme consequence des efforts hyperkinétiques de Гestomac! Or il n'y a jamais de stase gastrique dans cette affection, et on ne comprend guère comment le sphincter, spasmé, autoriserait le glissement des plis à travers sa lumière rétrécie. Au contraire, le pylore est largement ouvert (Nygaard et coll. insistent à 